\title{
The filing of bacterial plaque on orthodontic appliances: type of bracket and motivational strengthening
}

\author{
Rita Politangeli ${ }^{1}$ \\ Silvia Sabatini ${ }^{1}$ \\ Gianna Maria Nardi ${ }^{1}$ \\ Roberto Di Giorgio² \\ Gabriella Galluccio ${ }^{3}$
}

\begin{abstract}
${ }^{1}$ Master in Advanced Technologies for the Oral Hygiene, "Sapienza" University of Rome, Rome, Italy ${ }^{2}$ Course for Oral Hygiene Degree - B, "Sapienza" University of Rome, Rome, Italy

3Post Graduate School in Orthodontics, "Sapienza" University of Rome, Rome, Italy
\end{abstract}

Corresponding Author:

Rita Politangeli

"Sapienza" University of Rome,

Via Caserta 6,

00161 Rome, Italy

E-mail: ritapolitangeli@libero.it

\begin{abstract}
Introduction: the purpose of an orthodontic treatment is the achievement of an ideal occlusion through the displacement of the teeth in the bone structure. The presence of bacterial plaque can affect the outcome, and is a possible damage to the periodontal tissues. The aim of this work is to check the time evolution of the plaque of both self-ligating and conventional edgewise orthodontic multibrackets appliances and verify the use of an orthodontic Diary as a motivational aid to improve the maintenance of a good level of oral hygiene at home.

Materials and methods: 30 patients with fixed orthodontic appliances (Damon 3 system, MBT system) were examined; of these 24 completed the study. The test performed examined in each patient 5 dental elements: of these 3 bonded with bracket, as study sites, and 2 without brackets, used as control sites. At the control appointments, at time to, $t 1, t 2$, the presence of evident bacterial plaque layer was evaluated in order to determine in which way the presence of plaque is due to the type of bracket and to the type of orthodontic arch in the two different orthodontic appliances. The data have been analyzed with descriptive statistics.

Results: a statistical significant difference was evident only for the reduction of the number of subjects positive to the presence of plaque after the use of the motivational tool. No statistical signifi-
\end{abstract}

cant difference was evident between the two different appliances.

Discussion: the presence of plaque is evident in both the appliances but with greater deposit deeper in the slot of the self-ligating orthodontic brackets. The use of the orthodontic diary contributes to increase the patient compliance.

KEY WORDS: orthodontics, oral hygiene, self-ligating brackets.

Introduction

Many studies have evaluated the effects of fixed orthodontic appliances on the oral bacterial flora and the resulting periodontal status $(1,2)$; however, only a few have evaluated the tying system as an additional factor $(3,4)$. Conventionally, to connect the orthodontic arch to the bracket, 3 different methods can be used: steel metallic ligatures, elastomeric ligatures and locking systems of the slot, in the case of self-ligating brackets (5-7).

Based on an evaluation of the effects of the orthodontic treatments, all these methods show advantages and disadvantages, but, about the retention of the bacterial biofilm, the literature suggests that the elastic ligatures should increase the retention of the biofilm to a greater extent than the two other methods. In fact, it is recommended to avoid the use of elastic ligatures in patients with poor oral hygiene, to avoid the risk of increased susceptibility to caries and gingivitis $(1,8,9)$. It is imperative, therefore, that the orthodontic patient must be constantly taken in care, during therapy, with appropriate instruction and motivation. The dental hygienist suggests methods and tools, usually with the formulation and implementation of individual protocols (10-12).

One of the most frequent problems is the finding of lack of commitment of the patient to the prescribed procedures for the maintenance of oral hygiene. It is always necessary to emphasize to patients the benefits of a good oral maintenance for not prolong treatment times and to not suffer of caries or periodontal damage. It is therefore useful to use every motivational aid such as the use of an orthodontic diary, where the patient can find all useful information on the management of dental hygiene at home and important notes on the orthodontic therapy and on oral hygiene practices in the orthodontic patient (13).

The aim of this work was therefore to assess the level of oral hygiene maintaining in patients wearing orthodontic appliances, both conventional and self ligating, 
resorting to a motivational journal of orthodontics. It has been therefore evaluated the reduction of the presence of plaque and dental calculus on the buccal surface and inside the slot of the orthodontic brackets, and the presence of bleeding before and after use of the aid in motivation.

\section{Materials and methods}

Thirty patients were selected among the patients treated at the Unit of Orthodontics, Department of Oral and Maxillo Facial Sciences of "Sapienza" University of Rome, 17 of which were bonded with conventional orthodontic appliances, and 13 with self-ligating appliances.

Criteria for inclusion in the sample of the study were age, between 12 and 16 years, dental relationship, as molar right and left first class, the presence of orthodontic appliance in both jaws, positioned in a range of time between 6 and 12 weeks before the date of the first observation, the absence of previous orthodontic treatment with fixed or removable appliances and the absence of systemic diseases and syndromes.

Therefore exclusion criteria have been: patient not entering for age and occlusal characteristics in the inclusion criteria, the presence of systemic or syndromic illnesses and orthodontic therapies started by a period superior to how much previously stated.

Dental elements 1.1, 1.6, 2.6, 3.6 and 4.6 have been the subject of observation. Data were collected on the presence of plaque and dental calculus on the buccal surface of the teeth, considered in the gingival point of the teethbracket interface, and inside the slot (Fig. 1), adopting an evaluation graded in: 0 , no deposit, and 1 , presence of deposit; the possible presence of bleeding has been evaluated with the BOP, bleeding on probing index.

The presence of deposits, even on only one of the five dental elements taken into consideration, has been considered as equal to 1 , while only the condition of complete absence of deposits on all the elements considered entailed a score of 0 . For this purpose an ex-

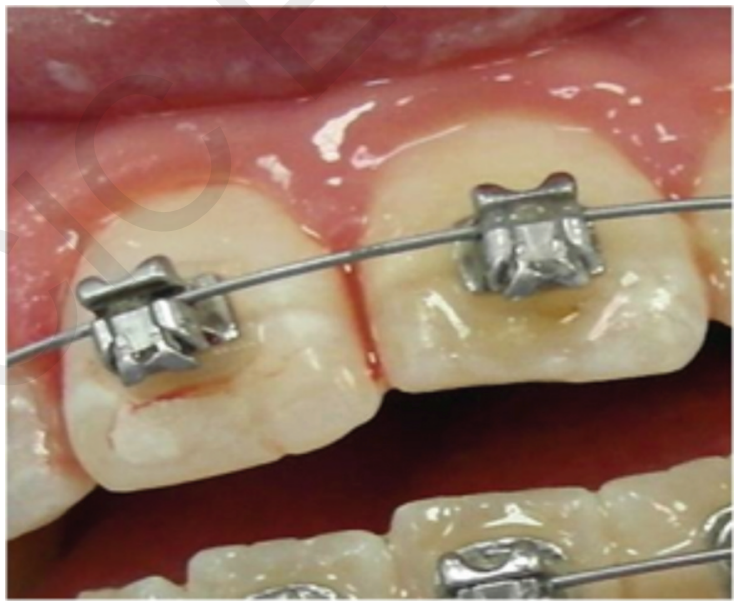

Figure 1 - The presence of bacterial plaque is evident inside the slot and on the buccal surface of the 1.1 tooth. The 2.1 tooth has been cleaned with an air flow device. plorer "probe CP15" was used (Figs. 2, 3) for the collection of the plaque, for the probing and for the evaluation of the bleeding.

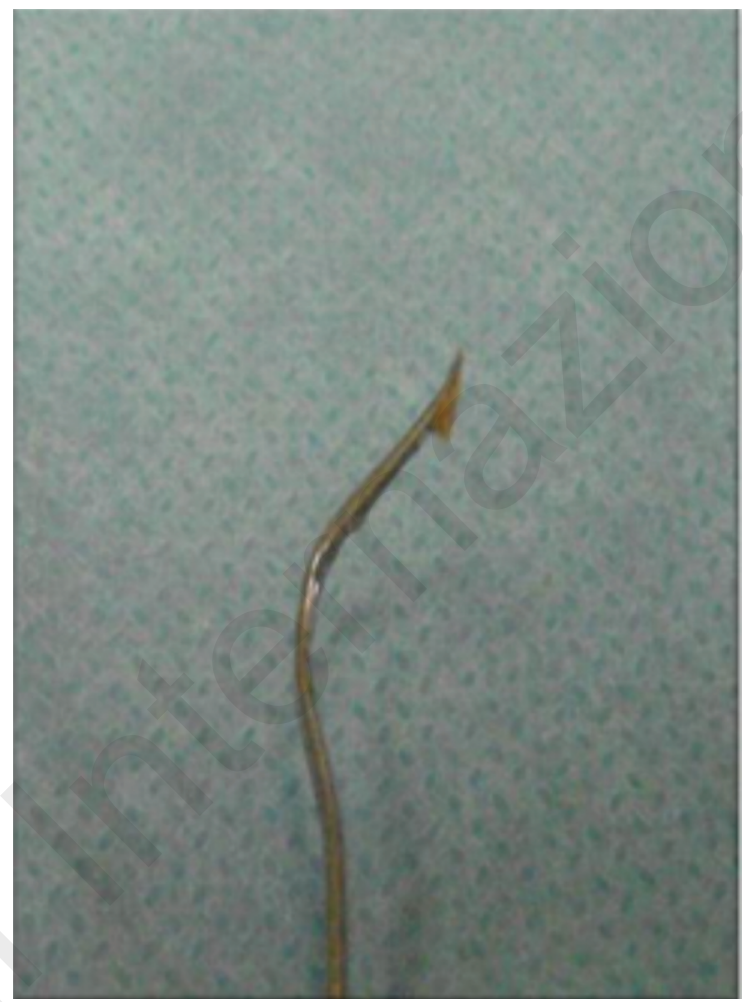

Figure 2 - Probe used for checking the presence of plaque.

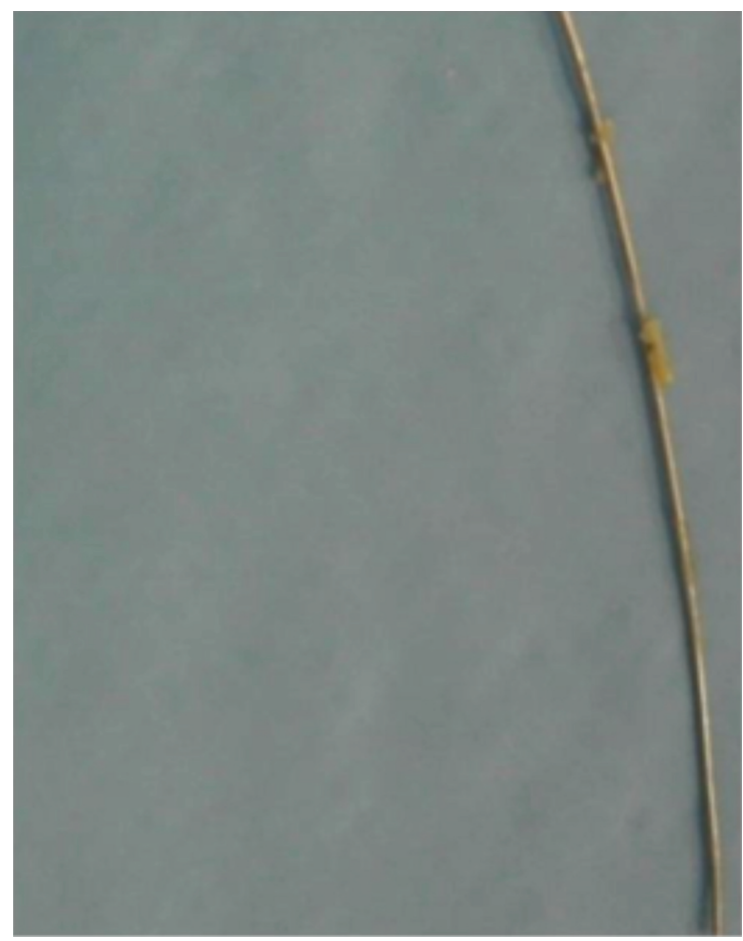

Figure 3 - Presence of bacterial plaque on the archwire in the areas corresponding with the self-ligating closure device. 
In order to evaluate the evolution during the time of the presence of plaque the test was repeated at three different times: T0, T1, T2.

- T0 (start of test) examination of the five elements during the first observation, at a distance of 6-12 weeks after bonding session. At this time the patients were all subjected to professional oral hygiene and were instructed to maintain hygiene at home.

- T1 (after 30 days): monitoring and recording of selected indices without removing the orthodontic archwires, repetition of the oral hygiene instruction but not of the professional oral hygiene and delivery of orthodontic diary (Fig. 4).

- T2 (after 60 days): tracking and recording of selected indices. In addition to the checks carried out at TO and T1, removing of the orthodontic arches and recording the presence of plaque and dental calculus on the archwire and inside the slot.

Of the initial group of patients, only 24 subjects completed the planned controls selected for the study, with a result of a final sample of 24 patients, of whom 16 with conventional appliances and 8 with self ligating appliances (mean age 13,6 $\pm 1,14 \mathrm{~F}, 10 \mathrm{M}$ ) (Fig. 5).

All observations concerning the presence of plaque and calculus were performed by two experienced operators, and in 5 patients, randomly selected among the group, the measurements were repeated by both operators, then applying the formula of Dhalberg for the evaluation of the method error, with a not significant result.

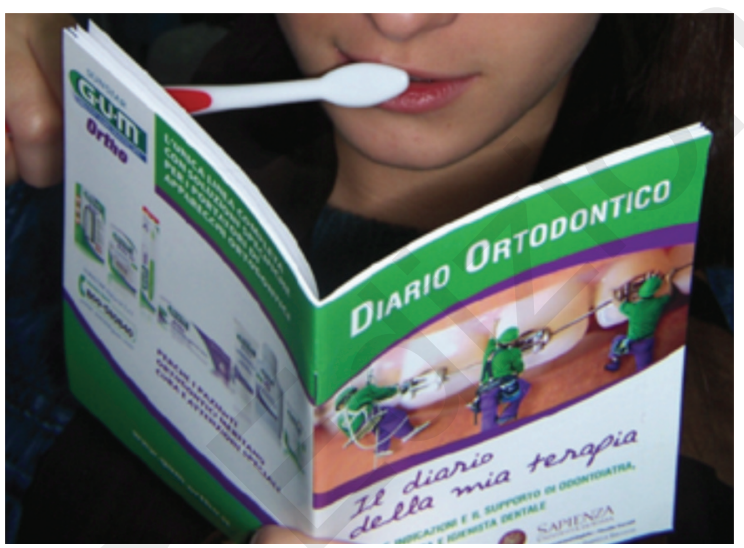

Figure 4 - The orthodontic diary.

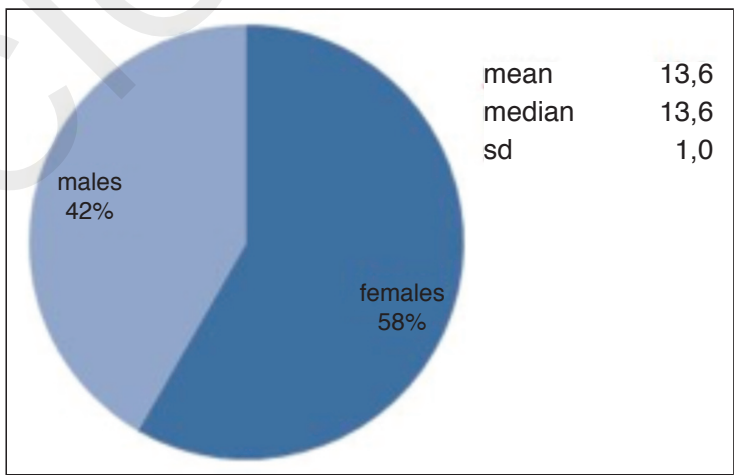

Figure 5 - Age/gender distribution of the population.
The collected data were then subjected to descriptive statistical analysis, mean and SD for the characteristic of the sample, and percentage of patients positive or negative to the check of dental plaque presence for each time of control, while the possible presence of differences between before and after the use of the orthodontic diary was performed with the Wilcoxon test.

\section{Results}

The results obtained are summarized in Table 1. In a first observation, recorded before the placement of the brackets, 6 of 15 patients were observed with the presence of plaque on the buccal surface. These data were only recorded as usual in the patient's monitoring in the orthodontic Unit but were not included in the study. After proper oral hygiene instructions, given uniformly to all patients, at time TO was possible to find only the occasional occurrence of dawn matter, but not presence of bacterial plaque.

Observations at time T1 were performed without removing the archwire and, among patients with self-ligating brackets, 4 of 8 were found to have, in the vestibular areas, the presence of plaque, whereas in patients treated with conventional brackets was found the presence of buccal plaque layer in 13 of 16 patients.

At time T2 the control of the parameters proceeded, also removing the orthodontic arches, and the observation of the presence of any deposits both on the arches and in the slot was added. It was found that only 1 of the patients, treated with self-ligating brackets, presented material, plaque or dental calculus in the vestibular areas, probably thanks to the morphology and reduced size of the brackets, but showed, vice ver$\mathrm{sa}$, in all of them, the presence of plaque and dental calculus internal to the slot, and this is probably due to the inability of the patient to clean inside these spaces, closed by the locking system doors.

The obtained data regarding conventional brackets resulted opposites: the presence of plaque buccally was found in 9 of 16 patients, whereas the slots presented without deposits, with the exception of the presence, in 2 of 16 patients, of limited quantity dawn matter.

The differences between the observations carried out during checks at time T1, before use of orthodontic journal, and the time T2, following the instructions for the correct compilation and use of orthodontic journal, show statistically significant differences for the values of presence of plaque, for $p<0.016$ for both groups (Tab. 1).

Table 1 - Subjects positive to the presence of plaque: percentages and statistical significance.

\begin{tabular}{lllll}
\hline $\begin{array}{l}\text { Pres. } \\
\text { of plaque }\end{array}$ & bonding & $\mathbf{3 0}$ days & $\mathbf{6 0}$ days & Wilcoxon t. \\
\hline & T0 & T1 & T2 & \\
\hline 0 & 24 & 7 & 14 & \\
1 & 0 & 17 & 10 & \\
& $0 \%$ & $81,25 \%$ & $56,25 \%$ & $\mathrm{P}<0.016$ \\
\hline
\end{tabular}


It was not possible to assess the difference separately for the two treatment groups because the size of the two samples resulted to be too small. The presence of plaque on the buccal surface of patients bonded with conventional brackets was however correlated with the presence of an increase bleeding of probing in these patients.

\section{Discussion}

In recent decades, the number of orthodontic treatments have increased consistently, as many patients recur to these therapies in order to improve the aesthetic appearance, while only a minority requires this type of treatment for health reasons $(5-7,14)$. Current progress in the field of orthodontics have made it possible to add to the traditional orthodontic approach the self-ligating appliances, that have the unquestionable advantage to reduce the friction between this system composed of archwire, bracket and ligature $(14,15)$. In order to try to overcome the limitations imposed by the frictional forces, industrial research has turned its attention to the interaction archwires - slots in presence of tied ligatures; these ligatures could be a brake for orthodontic movements. The result of these researches have been followed by the introduction on the market of new products: low friction conventional ligatures and self-ligating brackets. In this type of brackets reduced friction is often cited as a primary advantage compared to conventional brackets (2).

The reduction of friction in self-ligating brackets should be able to produce a more continuous and physiological tooth movement, ideally without exceeding, in the levels of exerted force, the capillary pressure, with the final effect of a reduction of the treatment time, decrease chair time, better infection control, less patient discomfort and better oral hygiene $(2,14)$. In fact, without an accurate prevention program, before, during and after orthodontic treatment, the likelihood of developing caries increases, also in relation to the lenght of the orthodontic treatment $(6,9)$.

The reduction of macroscopically evident plaque deposit in the sample analyzed during the observational period, before and after the administration of hygiene instructions and use of the orthodontic diary, also if the sample size was small, enhance the need of a self control of the compliance. The proper control of the oral hygiene is so demonstrated to be a consequence of the personal involvement and empowerment of the patient. We can consider that it is therefore essential to establish an individualized prevention program that includes a phase of education and motivation of the patient and a timetabled control plan, according to the needs of the patient, asking for a greater commitment to oral hygiene procedures.

The composition of the bacterial flora, indeed, is affected by the application of orthodontic appliances and the incidence of periodontitis increases with time $(9,16,17)$. The reason for this study was to find information on the accumulation of bacterial plaque in patients with orthodontic brackets of conventional and self-ligating type, both outside and inside the slots. From the analysis made on the results obtained it has been shown, in agreement with what has already been highlighted in the literature, that brackets with conventional ligatures make difficult the maintenance of a good oral hygiene, resulting in an accumulation of plaque and in a significantly increased risk of decalcification of the enamel, while the self-ligating brackets, instead, allow an effective cleaning of the buccal surface, because of the reduced dimensions of the brackets and less need of auxiliary devices, and a great respect of the periodontal tissues, evident in a low value of $\mathrm{BCl}$ (bracket comfort index) (2).

Conversely, in the results of our study, even if on a small study sample, it is clear that the closed locking system for the slot does not allow to properly maintain them free from deposits; therefore remains to verify what impact would have the presence of deposits, especially if hard, with the expected decrease in friction. The presence of plaque and calculus could then cancel the possible advantages of friction reduction, specially searched in the use of self ligating systems; in this case the hygiene vigilance could comprehend a maintenance protocols with systematic removal of archwire and cleaning of the slot at each periodical control.

The need of a complete engagement of the archwire in the slot, in the final phases of treatment, together with the friction free sliding movement typical of the self ligating appliance, require the maximum of compliance from the patient in the constant application of the instructions of plaque removal. Both the orthodontist and the hygienist must strength the motivation capability in this direction.

The use of methods of motivation, education and verification by means of oral hygiene aids delivered to the patient, as the tested orthodontic diary, allows you to keep the attention of the patient focused on the oral hygiene, limiting significantly the formation of plaque and then conditioning the success of orthodontic treatment. The results of the study show an efficient empowerment effect using a recording tool able to make an enhancement of correct behavior. It seems also that the mainteinance protocols must be differentiated according to the different kind of orthodontic appliance, because of the different spatial position of the plaque deposit.

\section{References}

1. Ciancio SG. A comparison of Plaque Accumulation in bond versus banded teeth. J Dent Res. 1985; 64:359.

2. Marshall SD, Currier GF, Hatch NE, et al. Ask us. Self-ligating bracket claims. Am J Orthod Dentofacial Orthop. 2010; 138(2):128-131.

3. Garcez AS, Suzuki SS, Ribeiro MS, et al. Biofilm retention by 3 methods of ligation on orthodontic brackets: a microbiologic and optical coherence tomography analysis. Am J Orthod Dentofacial Orthop. 2011; 140(4):e193-198. doi: 10.1016/j.ajodo.2011.04.019

4. Pandis N, Papaioannou W, Kontou E, et al. Salivary Strptococcus mutans levels in patients with conventional and self-ligating brackets. Eur J Orthod. 2010; 32:94-99. 
5. Bishara Samir E. Ortodonzia Moderna, Delfino Antonio Editore, Roma, 2006.

6. Proffit WR, White RP, Sarver DM. Trattamento delle Deformità Dentofacciali, Elsevier-Masson, Milano, 2004.

7. Proffit WR, Fields HW, Sarver DM. Ortodonzia Moderna, Elsevier- Masson, Milano, 2008.

8. Noble J, Cassolato S, Karaikos N, Wiltshire WA. Point of care. Preventive and interceptive measures for improving and maintaining good oral hygiene and cariogenic control in orthodontic patients. J Can Dent Assoc. 2009; 75(6):441-453.

9. Saloum FS, Sondhi A. Preventing enamel decalcification after orthodontic treatment. J Am Dent Assoc. 1987; 115 (2):257-261.

10. Guastamacchia C, Ardizzone V. Igiene Orale Domiciliare, Presidi Tecniche Motivazioni, Elsevier-Masson, Milano, 2001.

11. Guastamacchia C, Ardizzone V. Igiene Orale Professionale, Prevenzione e Igiene Dentale, Elsevier Masson, Milano, 2001.
12. Wilkins EM. La Pratica Clinica dell'Igienista Dentale, Piccin, Padova, 2010.

13. Nardi GM, Sabatini S, Politangeli R, Galluccio G. Efficacia motivazionale del diario ortodontico nella gestione dell'igiene orale del paziente con ortodonzia fissa o mobile. Doctor Os. 2013; 24(8):763-767.

14. Bagden A, Chimenti C, Christensen Lars R, et al. The phenomenon of low friction techniques: state of the art, Ariesdue, Milano, 2006.

15. Björn L, Glasl B, Cacciafesta V. Bracket Autoleganti in Ortodonzia, Principi Biomeccanici e Applicazioni Cliniche, Griffin, Como, 2010.

16. Carranza Fermin A. Parodontologia clinica, Delfino Antonio Editore, Roma, 2003

17. Ramaglia L, Sbordone L, Ciaglia RN, et al. Un confronto clinico della efficacia ed efficienza delle due procedure di profilassi professionale in pazienti ortodontici. Eur J Orthod. 1999; 21(4):423-428. 